\title{
Aerosol Matrix-Assisted Laser Desorption Ionization Mass Spectrometry
}

\author{
Kermit K. Murray and David H. Russell \\ Department of Chemistry, Texas A \& M University, College Station, Texas, USA
}

\begin{abstract}
A new method of liquid sample introduction for a time-of-flight mass spectrometer (TOF-MS) has been developed by applying the method of matrix-assisted laser desorption ionization to aerosols. Analyte biomolecules are dissolved in a methanol solvent along with a UVabsorbing matrix and formed into an aerosol with a pneumatic nebulizer. The aerosol particles are dried in a heated skimmer tube before ionization by pulsed 355-nm UV laser radiation. Mass analysis is achieved in a linear TOF-MS. Results for the ionization of bovine insulin (5733.5 Mw) are reported. (J Am Soc Mass Spectrom 1994, 5, 1-9)
\end{abstract}

S ince its introduction, matrix-assisted laser desorption ionization (MALDI) has become one of the most useful mass spectrometric techniques for the molecular weight determination of high mass biomolecules [1-3]. In the typical MALDI experiment, the analyte biomolecule is deposited from solution onto a metal surface along with a $100-$ to $50,000-\mathrm{M}$ excess of a suitable matrix [4]. The solvents are allowed to evaporate and the sample is inserted into the source region of a mass spectrometer. Light from a pulsed laser operating in either the infrared (IR) [5] or UV [1] region is absorbed by the matrix, causing both ablation of the surface and ionization of the intact biomolecule. As it is commonly implemented, MALDI has two limitations. First, sample preparation is somewhat of an art and often not reproducible from preparation to preparation or even from position to position on the sample $[6,7]$. Second, manual sample preparation precludes the on-line use of MALDI with highperformance liquid chromatography (HPLC). These limitations could potentially be overrome with a suitable MALDI liquid introduction method.

Several research groups are working on novel approaches for liquid sample introduction with MALDI. One of the first reports for laser desorption of proteins in the mass range over $10 \mathrm{kDa}$ used a finely ground cobalt powder in glycerol [2]. Therefore, it should be feasible to carry out MALDI from solutions, provided matrices can be found. The commonly used fast-atom bombardment (FAB) matrices, glycerol, triethanolamine, and lactic acid, have been used with IR laser desorption [5-8] and 2-nitrophenyl octyl ether has been used with UV MALDI [1]. The most widely used liquid MALDI matrix, 3-nitrobenzyl alcohol, has been used with UV laser desorption in both static [1, 9-11]

Address reprint requests to Professor David H. Russell, Department of Chemistry, Texas A \& M University, College Station, TX 77843-3255. and continuous flow MALDI [12]. Two-step ionization methods using liquid volatilization followed by multiphoton ionization have been developed with IR laser desorption [13, 14] and heated pulsed valve [15] liquid introduction. Frozen aqueous matrices have been used with both static [16] and flowing [17] sample introduction. Aerosols have been used for sample introduction for single aerosol particle laser desorption ionization (LDI). McKeown et al. [18] have developed an apparatus for analysis of single aerosols by 193-nm LDI followed by linear time-of-flight mass spectrometry (TOF-MS) detection. Sinha [19] has developed a similar single aerosol particle LDI apparatus that uses 1064-nm laser ionization in conjunction with a focal plane mass spectrograph.

We describe an apparatus that combines aerosol sample introduction with MALDI-TOF-MS. Aerosols provide a consistent and constantly renewed surface for laser ablation. The MALDI sample is "prepared" when the aerosol is introduced into the vacuum and dried by solvent evaporation. Problems with reproducibility that occur with sample deposition by solvent evaporation on a metal surface are avoided. Aerosol MALDI occurs without an underlying surface and thus provides an opportunity to study the ionization mechanism without possible substrate effects. Aerosol MALDI is also compatible with mass spectrometric analysis of HPLC effluent at 0.5 to $1 \mathrm{~mL} / \mathrm{min}$ flow rates and when combined with TOF mass analysis provides a method for rapid liquid chromatography/mass spectrometry (LC/MS) detection of biomolecules. In our apparatus, aerosols are formed using a continuous pneumatic nebulizer from a methanol solution acidified with trifluoroacetic acid (TFA) and containing the analyte and matrix. The solvent is removed by passing the aerosol through a heated tube to form a collimated beam of dried aerosol particles. Ions are formed by pulsed UV laser radiation and mass se- 
lected in a linear TOF-MS. In a previous article preliminary aerosol MALDI results were presented [20]. In this work, a detailed description of the apparatus is given and some of the operational parameters are discussed. The aerosol MALDI mass spectrum of bovine insulin is presented and the relative ion yield is reported under various experimental conditions.

\section{Experimental}

A schematic of the aerosol TOF-MS is shown in Figure 1. Aerosols are produced by a pneumatic nebulizer with nitrogen carrier gas. The aerosol beam is skimmed to remove excess carrier gas and collimate the beam. Ions are produced by irradiating the aerosol beam with the output of a pulsed laser. The ions are accelerated into a linear TOF tube and detected with a microchannel plate particle multiplier.

The pneumatic nebulizer is depicted in Figure 2 and consists of three concentric stainless steel tubes. The central tube is a 1/16-inch tube with a 0.010 -inch bore (1.6-mm outer diameter, $250-\mu \mathrm{m}$ inner diameter) and supplies the liquid to the nebulizer. The central capillary tube protrudes $2 \mathrm{~mm}$ from a $3 / 16$-inch tube (4.8$\mathrm{mm}$ outer diameter, 3-mm inner diameter) through which the $\mathrm{N}_{2}$ carrier gas flows. Two short pieces of

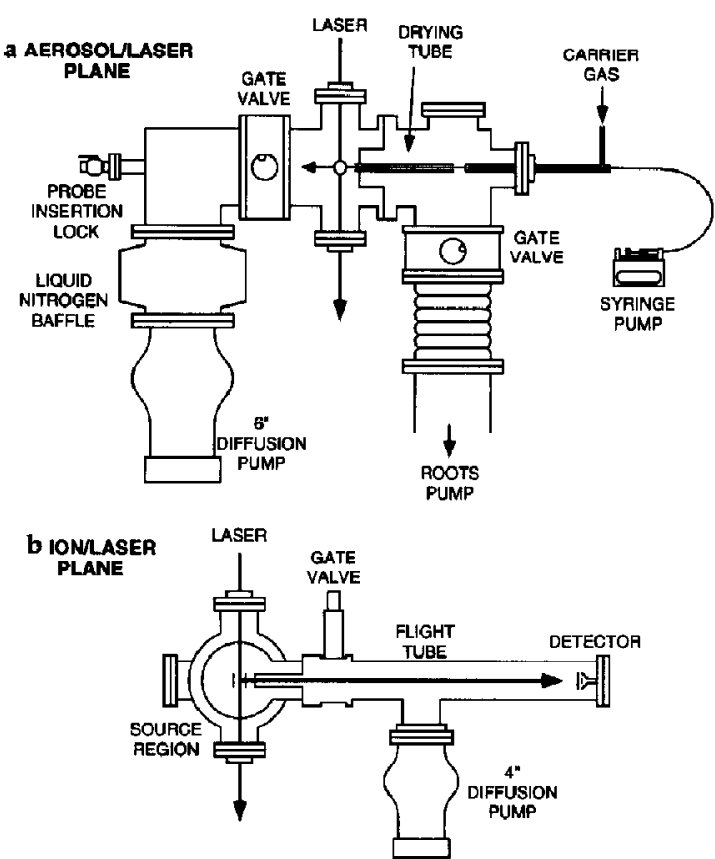

Figure 1. Aerosol MALDI TOF-MS. (a) View in the aerosol and laser beam planes. Aerosols are produced continuously in the first chamber and sent through a heated tube to the second chamber for ionization. The path of the ions is into the plane of the page. (b) View in the plane of the laser beam and ion flight path. The path of the aerosol beam is into the plane of the page. A pulsed laser creates ions that are accelerated for mass separation in a $1.1 \mathrm{~m}$ flight tube.

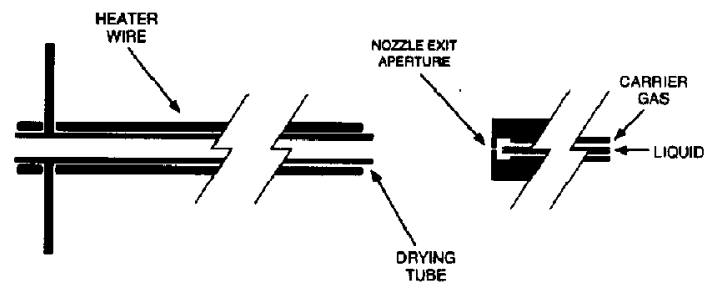

Figure 2. Detail of the nebulizer and drying tube. Nitrogen gas flows around the central tube, producing an aerosol from liquids drawn through the $250-\mu \mathrm{m}$ capillary. The nozzle exit aperture is $1 \mathrm{~mm}$ in diameter. Aerosols flow through a drying tube $25-\mathrm{cm}$ long with a $4-\mathrm{mm}$ inner diameter wrapped in heating wire and heated to $300{ }^{\circ} \mathrm{C}$.

wire spot welded to the tip of the $3 / 16$-inch tube support and center the capillary tube. A 1/2-inch tube (13-mm outer diameter) holds the $70-\mathrm{cm}$ long assembly, which is inserted into vacuum through a $1 / 2$-inch fitting. An aluminum end cap with a $1-\mathrm{mm}$ hole screws into the tip of the $1 / 2$-inch tube to form the aerosol nozzle exit aperture. A syringe pump forces the liquid solution through the rentral tube at a flow rate of 0.5 $\mathrm{mL} / \min (8 \mu \mathrm{L} / \mathrm{s})$. The liquid flows to the tip of the central tube where it is nebulized and entrained in the carrier gas flow and forced through the nozzle exit aperture. The aerosol forms a cone of approximately $15^{\circ}$ half angle that can be observed by illumination with bright incandescent light or by scattered $633-\mathrm{nm}$ helium-neon laser radiation. The aerosol formation chamber is evacuated by a $330 \mathrm{~L} / \mathrm{s}$ roots blower and rotary piston backing pump. Pressure in the acrosol formation chamber with the nebulizer operating is approximately 1 torr.

The aerosol beam is skimmed $2 \mathrm{~cm}$ downstream when it enters a 25 -cm long $1 / 4$-inch copper tube (4-mm inner diameter, 6-mm outer diameter). The nozzle-to-skimmer distance is adjustable and is optimized for the maximum throughput of aerosol while still maintaining adequate vacuum in the ion formation and TOF regions. The copper tube serves two functions: (1) to act as an aerosol beam skimmer and collimate the beam by allowing only the central portion to enter the ionization chamber, and (2) to serve as an aerosol drying tube. To promote solvent evaporation, the tube is wrapped in fiberglass-insulated nickel-chromium wire to which a current is applied. A thermocouple wire located under the heater wire at the tube midpoint is connected to a thermostatic controller, which regulates temperature. The experiments described below were obtained at a drying tube temperature of $300{ }^{\circ} \mathrm{C}$.

After exiting the skimmer/drying tube, the aerosol particles drift for $2.5 \mathrm{~cm}$ before crossing a pulsed laser beam at a $90^{\circ}$ angle. For the work described here, the $10-\mathrm{Hz}$ frequency tripled output of an Nd:YAG laser at $355 \mathrm{~nm}$ is used. A $25-\mathrm{cm}$ focal length spherical lens is used to focus the laser to a spot size of 0.5-mm diameter at the laser/aerosol beam intersection. The diame- 
ter of the aerosol beam at this point is $5 \mathrm{~mm}$, giving an interaction volume of $10^{-3} \mathrm{~cm}^{3}$. A typical laser pulse energy is $1 \mathrm{~mJ}$, resulting in a fluence of $0.5 \mathrm{~J} / \mathrm{cm}^{2}$ and an irradiance of $80 \mathrm{Mw} / \mathrm{cm}^{2}$ given the 6-ns laser pulse duration. The interaction volume is equidistant between the repeller plate and the extraction grid of the TOF-MS with the ion flight path perpendicular to both the aerosol and laser beams. The repeller plate and extraction grid are circular disks $5 \mathrm{~cm}$ in diameter separated by $2 \mathrm{~cm}$, with the latter having a central $1-\mathrm{cm}$ hole covered by a $90 \%$ transmittance, 70 line/inch grid through which ions pass into the TOF tube. The repeller plate is held at a potential of $5 \mathrm{kV}$ and the extraction grid is held at ground. The ion formation chamber is evacuated by a liquid nitrogen-trapped $2400 \mathrm{~L} / \mathrm{s}$ 6-inch diffusion pump, which produces a pressure of $1 \times 10^{-4}$ torr with the nebulizer running.

The ions enter the flight tube through a $5-\mathrm{mm}$ diameter aperture $1 \mathrm{~cm}$ beyond the extraction grid. The flight tube is evacuated by an unbaffled $1200 \mathrm{l} / \mathrm{s}$ 4 -inch diffusion pump giving a pressure of $1 \times 10^{-5}$ torr with the nebulizer running. Ions are detected at the end of the 1.1-m flight path with a microchannel plate detector. The side of the microchannel plates facing the flight tube is held between -2.0 and -2.5 $\mathrm{kV}$ with the opposite side held between -250 and $-300 \mathrm{~V}$. A grid $1 \mathrm{~cm}$ away from the plates keeps the flight tube field free. The detector signal is sent to the $50-\Omega$ input of a digital oscilloscope (LeCroy 9400 or 9450) triggered by the laser Q-switch synchronization output signal. Mass spectra are averaged over 1000 to 2000 shots by the oscilloscope and then downloaded to an IBM-compatible, 80386-based microcomputer for analysis.

Typical data manipulation consists of summing 5 to 10 successive data points to improve signal to noise. For example, a data set of 25,000 data points with a 20-ns separation between points can be reduced to a data set of 5000 points with a 100-ns separation between points. Mass calibration is performed based on assignments of peaks in the mass region below $300 \mathrm{Da}$. Peak positions, areas, and widths are determined by a nonlinear least squares fit to the data using a gaussian peak contour.

Solid aerosol deposited samples can be collected on the tip of a probe inserted through a vacuum insertion lock into the ionization chamber. The deposited solid samples are removed from the vacuum for inspection under an optical microscope. Optical microscopy is performed on a Zeiss Axiophot compound optical microscope equipped with a $35-\mathrm{mm}$ film cartridge. Solid samples are prepared by flowing 1 to $3 \mathrm{~mL}$ of solution through the nebulizer and collecting the deposit at the point of laser and aerosol beam intersection.

Solutions were prepared by dissolving both matrix and analyte in methanol acidified with TFA. Matrix compounds used were 4-nitroaniline 99\%, 3,5-dimethoxy-4-hydroxy cinnamic acid (sinapic acid) $98 \%$, and $\alpha$-cyano-4-hydroxy cinnamic acid $97 \%$ (Aldrich
Chemical Co., Milwaukee, WI), 2-cyano-4-nitroaniline 98\% (Fluka, Ronkonkoma, NY), and 4-nitrophenol, 7 hydroxy-4-methyl coumarin (4-methylumbelliferone, Sigma Chemical Co., St. Louis, MO). Bovine insulin (Sigma) was used with no further purification. The solvents used were anhydrous $99.8 \%$ spectrophotometric grade methanol (Mallinckrodt, Paris, KY) and TFA (Sigma).

\section{Results}

This article focuses on several of the parameters influencing the performance of aerosol MALDI. Optical microscopy gives an estimate of the aerosol particle size under different formation conditions. The aerosol MALDI mass spectrum of bovine insulin is presented and the bovine insulin ion yield is shown under different experimental conditions. Experimental parameters discussed are the matrix-to-analyte ratio, performance of several different MALDI matrices under aerosol MALDI conditions, ion yield at threshold laser energy levels, and ion yield as a function of drying tube temperature.

An estimate of aerosol particle size was obtained from optical microscopy of aerosols deposited on metal surfaces inserted into the vacuum. Microscope photographs were obtained under different conditions of aerosol drying, and aerosol particle diameters were obtained from properly scaled photographic images. Various matrices and matrix/analyte combinations were used, but the observed aerosol particle sizes did not depend on the solutes used. Aerosol deposits made using a 6-cm drying tube gave aerosols with diameters of approximately $50 \mu \mathrm{m}$. The longer $25-\mathrm{cm}$ drying tube produced aerosols of approximately $10 \mu \mathrm{m}$ in diameter. Observations made with the $25-\mathrm{cm}$ drying tube indicate that the diameter does not vary with drying tube temperatures from 25 to $500{ }^{\circ} \mathrm{C}$, suggesting that contact with the desolvation gas at high pressures is more important than the temperature of the gas itself. Optical microscopy also gives an indication of the degree of solvation of the aerosols. Wet particles hitting the surface leave nearly concentric rings of solute, indicating both inilial particle diameter and evaporation of solvent after surface deposit. Additionally, resolvation and recrystallization of the matrix and analyte deposited on the surface indicate that wet aerosol particles are being deposited. We have found that under the conditions that give aerosol MALDI signal, at least some of the aerosols contain an appreciable quantity of solvent.

Bovine insulin was used as a test biomolecule for aerosol MALDI because it is inexpensive, easily obtained, and has been thoroughly investigated using a variety of desorption ionization methods [21-24]. Figure 3 shows a mass spectrum of bovine insulin in a 4-nitroaniline matrix with a 48:1 matrix-to-analyte molar ratio. The solution used for the mass spectrum in Figure 3 was $3.4 \times 10^{-4} \mathrm{M}$ in bovine insulin, $0.016 \mathrm{M}$ 


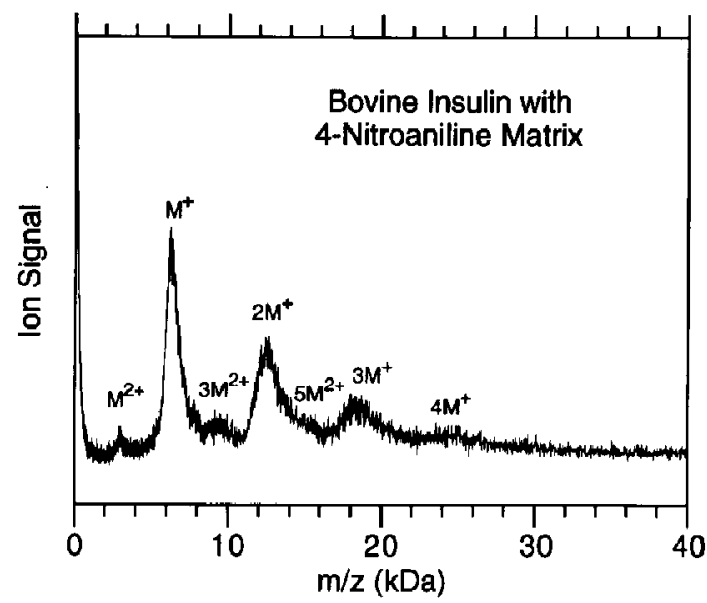

Figure 3. Aerosol MALDI mass spectrum of bovine insulin (5733.5 Mw) obtained with 4-nitroaniline matrix at a 48:1 $\mathrm{M}$ ratio. The spectrum was averaged over 2000 laser shots at a pulse energy of $1 \mathrm{~mJ}$.

in 4-nitroaniline, and $0.1 \mathrm{M}$ in TFA in a methanol solvent. The TOF acceleration voltage was $5 \mathrm{kV}$ and the laser pulse energy was $1 \mathrm{~mJ}$ at $355 \mathrm{~nm}$. Bovine insulin ion (denoted $\mathrm{M}^{+}$) and bovine insulin cluster ions as large as $4 \mathrm{M}^{+}$are seen as intense broad peaks in the spectrum. The notation $\mathrm{M}^{+}$here indicates an ion of a single bovine insulin molecule possibly protonated or cationized by $\mathrm{Na}^{+}$or $\mathrm{K}^{+}$and possibly clustered with one or more solvent or matrix molecules. The doubly charged ions $\mathrm{M}^{2+}, 3 \mathrm{M}^{2+}$, and $5 \mathrm{M}^{2+}$ are also observed. The temporal width of the bovine insulin $\mathrm{M}^{+}$peak is $9 \mu$ s full width at half maximum when fit to a gaussian peak contour. The mass resolution ( $m / \Delta m$ or equivalently $t / 2 \Delta t$ ) corresponding to this peak width is 6 if it is assumed that the peak results from a single ion. The measured positions of the bovine insulin and bovine insulin cluster ion peaks are 6300 for $\mathrm{M}^{+}, 12,700$ for $2 \mathrm{M}^{+}, 18,600$ for $3 \mathrm{M}^{+}$, and 24,800 for $4 \mathrm{M}^{+}$, with an accuracy of approximately $1 \%$ for the peak centers. These peak position values are 8 to $10 \%$ higher than expected for bovine insulin clusters and indicate either bovine insulin clustering with matrix and solvent or a systematic error in extrapolating the mass calibration from low to high mass-to-charge ratio.

Figure 4 shows the low mass portion of the spectrum for bovine insulin in a 4-nitroaniline matrix. The spectrum is shown at $20 \mathrm{~ns}$ / point with no data manipulation other than mass calibration. Initial mass calibration was based on the identification of $\mathrm{Na}^{+}$and $\mathrm{K}^{+}$ peaks present due to alkali contaminants in the solvent and samples. The dominant ions in the low mass region of the mass spectrum arise from a progression starting at mass 33 and separated by 32 mass units. These peaks are assigned to protonated methanol clusters of the form $\left(\mathrm{CH}_{3} \mathrm{OH}\right)_{n} \cdot \mathrm{CH}_{3} \mathrm{OH}_{2}^{+}$that are present due to incomplete desolvation of the aerosol. A strong

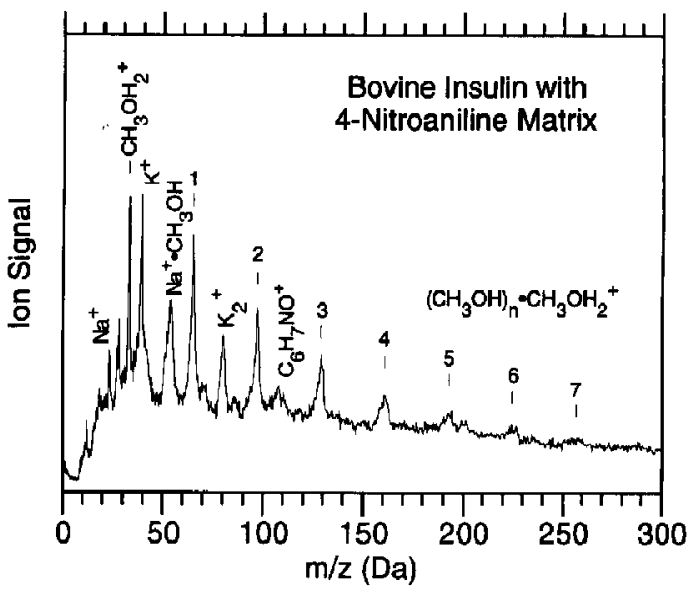

Figure 4. Low mass portion of Figure 3. The numbered peaks correspond to protonated methanol clusters of the form $\left(\mathrm{CH}_{3} \mathrm{OH}\right)_{n} \cdot \mathrm{CH}_{3} \mathrm{OH}_{2}^{+}$.

signal from sodiated methanol $\mathrm{CH}_{3} \mathrm{OH} \cdot \mathrm{Na}^{+}$at mass 55 is observed with the sodium arising from trace contamination in the solutions. The only peak that can be ascribed to the innization of matrix molecules is in the vicinity of $m / z 108$, which is assigned to the loss of NO from the 4-nitroaniline matrix. A strong peak at mass 109 has been observed in our laboratory with surface MALDI using a 4-nitroaniline matrix and is ascribed to the loss of NO from the protonated matrix [25]. Mass calibration for Figures 3 and 4 was performed by using the peaks corresponding to $\mathrm{Na}^{+}, \mathrm{K}^{+}$, $\mathrm{CH}_{3} \mathrm{OH} \cdot \mathrm{Na}^{+}$, and $\left(\mathrm{CH}_{3} \mathrm{OH}\right)_{n} \cdot \mathrm{CH}_{3} \mathrm{OH}_{2}^{+}$with $n=0$ to 6 . The mass resolution of the protonated methanol peaks is approximately 30 .

The concentrations of matrix and analyte used in the aerosol solutions are based on two considerations: the maximum matrix concentration that can be used without excessive nozzle clogging and the optimum matrix-to-analyte molar ratio. The maximum concentration of matrix that can be used is approximately 0.02 $\mathbf{M}$ and corresponds to several milligrams of matrix per milliliter of solvent. The optimum matrix-to-analyte ratio has been found to be the minimum possible [26]. This is in striking contrast to surface MALDI where the optimum matrix-to-analyte ratio is typically between 100 and 50,000 [4]. The optimum amount of analyte for use with aerosol MALDI is the maximum that can be dissolved in solution. For the peptides that have been used, several milligrams per milliliter is sufficient for both ion signal and nebulizer performance. The bovine insulin solution used to obtain the spectrum in Figure 3 was prepared using approximately $2 \mathrm{mg} / \mathrm{mL}$ each of bovine insulin and 4-nitroaniline, nearly the maximum amounts allowable for nebulization. The resulting matrix-to-analyte ratio of approximately $50: 1$ is the minimum obtainable under the above criteria. Note that the minimum matrix-to-analyte ratio will increase with 
increasing analyte molecular weight due to these solubility considerations.

Figure 5 shows a plot of the integrated $\mathrm{M}^{+}$peak area for bovine insulin as a function of laser pulse energy. A 4-nitroaniline matrix was used at a 50:1 molar ratio and the laser pulse energy was varied between 0.25 and $20 \mathrm{~mJ}$. This range of pulse energies corresponds to a fluence range of 0.1 to $10 \mathrm{~J} / \mathrm{cm}^{2}$ and an irradiance range of $20 \mathrm{Mw} / \mathrm{cm}^{2}$ to $1.6 \mathrm{Gw} / \mathrm{cm}^{2}$. The error bars for the $\mathrm{x}$ axis indicate long-term variation of approximately $10 \%$ in the laser pulse energy. The error bars for the $\mathrm{M}^{+}$ion signal are estimated from the signal-to-noise level and the variation in peak area obtained using various fitting methods. The data are plotted on a logarithmic scale and hence a straight line indicates a functional dependence of

$$
S=\alpha P^{n}
$$

where $S$ is the $\mathrm{M}^{+}$signal intensity, $P$ is the laser pulse energy, and $\alpha$ is a constant of proportionality. The data in Figure 5 show a steep increase in signal intensity near the threshold pulse energy with the steepness decreasing at higher energies. For pulse energies above $10 \mathrm{~mJ}$ the signal is nearly constant. The four lines in Figure 5 indicate pulse energy dependences of $n=1$ to 4 with the value of $\alpha$ chosen such that the lines intersect with the $n=1$ line at $P=1 \mathrm{~mJ}$. Below $1 \mathrm{~mJ}$ the data follow an $n$ dependence of 2 or 3 equally well, but show a nearly linear behavior at energies between 1 and $10 \mathrm{~mJ}$.

Figure 6 is a plot of the width and area of the bovine insulin $\mathrm{M}^{+}$peak as a function of the drying tube temperature. The maximum $\mathrm{M}^{+}$area is set to 1 for the purposes of the plot and the relative scaling of the two $\mathrm{y}$ axes is arbitrary. The matrix used was 4-nitroaniline at a 50:1 matrix-to-analyte molar ratio and the laser pulse energy was $4 \mathrm{~mJ}$. The drying tube

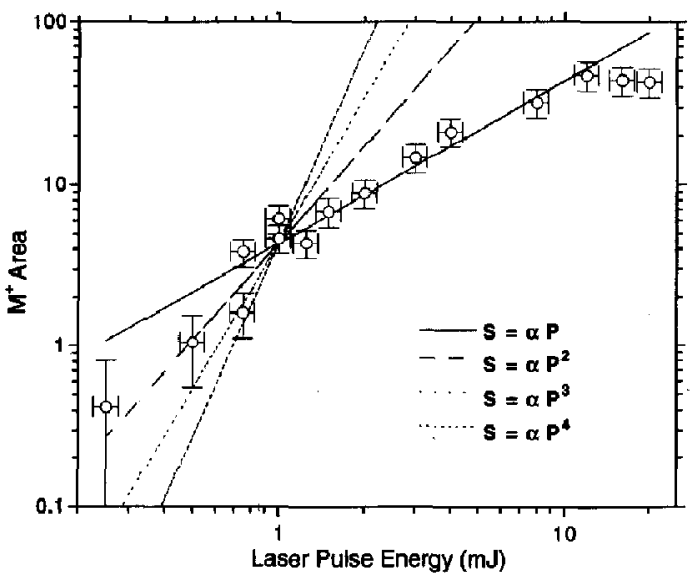

Figure 5. Log-log plot of laser pulse energy dependence of bovine insulin $\mathrm{M}^{+}$signal near threshold. The matrix was 4nitroaniline at a 50:1 $\mathrm{M}$ ratio.

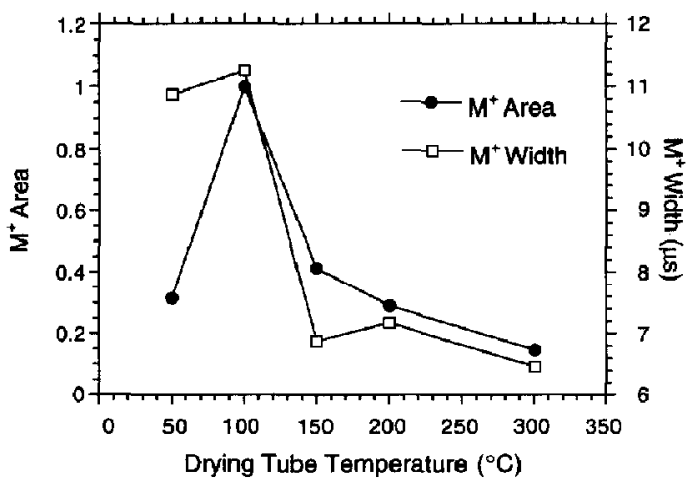

Figure 6. Bovine insulin $\mathrm{M}^{+}$peak area and width as a function of drying tube temperature. The matrix was 4-nitroaniline at a 50:1 M ratio.

temperature was varied between 50 and $300{ }^{\circ} \mathrm{C}$. The flight tube pressure increased with decreasing drying tube temperature reaching $5 \times 10^{-5}$ torr at $50{ }^{\circ} \mathrm{C}$, a level possibly detrimental to the microchannel plate detector if used for extended periods. The integrated bovine insulin $\mathrm{M}^{+}$ion signal increases steadily as the drying tube temperature is lowered to $100^{\circ} \mathrm{C}$ from the typically used $300^{\circ} \mathrm{C}$, followed by a sharp drop off between 100 and $50{ }^{\circ} \mathrm{C}$. The bovine insulin $\mathrm{M}^{+}$peak width follows a parallel trend to the peak area, increasing as the drying tube temperature is decreased. The width of the $\mathrm{M}^{+}$peak, however, stays relatively constant between 100 and $50^{\circ} \mathrm{C}$ while the peak area drops sharply.

Several different matrices were tested for aerosol MALDI of bovine insulin. The matrices used in addition to 4-nitroaniline were 2-cyano-4-nitroaniline, 3,5dimethoxy-4-hydroxy cinnamic acid (sinapic acid), $\alpha$ cyano-4-hydroxy cinnamic acid, 4-nitrophenol, and 7hydroxy-4-methyl coumarin. The solutions were mixed to give a 50:1 matrix-to-analyte ratio at a bovine insulin concentration of $3.0 \times 10^{-4} \mathrm{M}$ in methanol solvent acidified with $0.1 \mathrm{M}$ TF $\Lambda$. The laser pulse energy was $4 \mathrm{~mJ}$ and spectra were obtained as an average over 2000 laser shots. The integrated intensities of the bovine insulin $\mathrm{M}^{+}$peaks give an indication of the relative effectiveness for the ionization of bovine insulin. The $\mathrm{M}^{+}$intensity of 4-nitroaniline is set to 1 for the purposes of comparison. The results for the relative $\mathrm{M}^{+}$signal intensities are $\alpha$-cyano-4-hydroxy cinnamic acid: 12 ; 4-nitroaniline: 1; 2-cyano-4-nitroaniline: 0.2; 7-hydroxy-4-methyl coumarin: 0.06; 4-nitrophenol: 0.04; 3,5-dimethoxy-4-hydroxy cinnamic acid (sinapic acid): 0.02 . All of the matrices produce a detectable ion signal but the variation from the best ( $\alpha-$-cyano-4-hydroxy cinnamic acid) to the worst (3,5-dimethoxy-4-hydroxy cinnamic acid) is nearly three orders of magnitude. The matrix $\alpha$-cyano-4-hydroxy cinnamic acid gives the best signal but also the broadest bovine insulin peaks. The matrix 4-nitroaniline appears to be the best for bovine insulin under the conditions used because it 


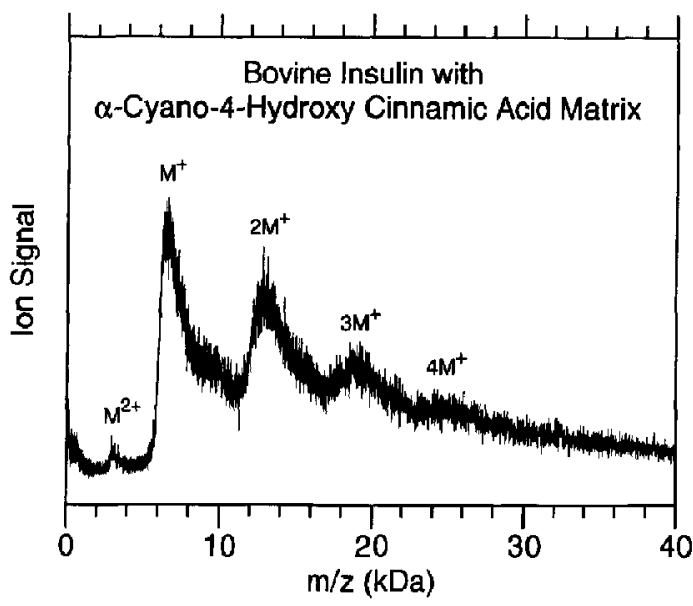

Figure 7. Aerosol MALDI mass spectrum of bovine insulin (5733.5 Mw) obtained with $\alpha$-cyano-4-hydroxy cinnamic acid matrix at a 50:1 $\mathrm{M}$ ratio. The spectrum was averaged over 2000 laser shots at a pulse energy of $1 \mathrm{~mJ}$.

provides a relatively intense ion signal without excessive peak broadening. As with surface MALDI, it is expected that the optimum matrix will be different for different analytes and experimental conditions. Figure 7 shows the spectrum of bovine insulin in a matrix of a-cyano-4-hydroxy cinnamic acid. The width of the rising edge of the $\mathrm{M}^{+}$peak gives a resolution of 6 , but the peak tails to the high mass side.

Although methanol has been used exclusively as the solvent for the studies described here, several other solvents have been used successfully with aerosol MALDI. We have used ethanol, acetonitrile, and mixed solvents such as water-methanol, acetonitrile-methanol, and water-acetonitrile-methanol. The less volatile solvents such as water and acetonitrile, when used by themselves or in high percentage mixtures, have a tendency to form ice at the tip of the skimmer that can eventually block the aerosol flow. Icing problems can potentially be overcome with modifications to the skimmer and drying tube assembly.

\section{Discussion}

\section{Comparison of Surface and Aerosol MALDI}

Surface MALDI is a well-established technique and it is instructive to make a general comparison between MALDI of aerosols and MALDI of solid surfaces. In both cases laser radiation is absorbed by the matrix causing ablation and analyte ionization, possibly by proton transfer from the matrix [25]. Aerosol MALDI differs from surface MALDI in the following: (1) its mass resolution is lower; (2) its limit of detection is higher; (3) its mass peaks in the low molecular weight region ( $<300 \mathrm{Da})$ arise from protonated and alkylated solvent clusters and not from ionized matrix molecules;
(4) a low matrix-to-analyte ratio is favored; (5) high laser irradiances give the best ion signal with no observable analyte fragmentation. Possible reasons for these differences are discussed below. Instrument improvements are also discussed, along with the outlook for aerosol MALDI as a detection method for $\mathrm{LC}$.

The mass resolution of the aerosol MALDI apparatus is influenced by several factors including the initial spatial, energy, and temporal spread of the ablated ions. In addition, the observed peaks can be broadened by clustering, collisions in the ion source region, or metastable ion decay. It is difficult to ascertain the energy and temporal spread of ions; however, spatial spread is determined by the apparatus geometry and can be easily estimated. Ions are created in a cylindrical interaction volume with a $0.5-\mathrm{mm}$ diameter equal to that of the laser beam and a 5 -mm length equal to the diameter of the aerosol beam. The calculated spread in time at the detector of singly charged bovine insulin ions that are created in this volume and accelerated into the flight tube is $6 \mu \mathrm{s}$. This value is close to the measured value of $9 \mu \mathrm{s}$ and is within the experimental error for the measured laser beam diameter. Additional contributions to peak widths due to clustering is a concern because it is apparent that the aerosol is not completely desolvated before ablation. Both optical microscopy and the low mass portion of the bovine insulin mass spectrum in Figure 4 indicate that solvent is present when the aerosols are ionized. It is highly likely that methanol is clustered to the bovine insulin as well. Metastable decay may also contribute to broadening and give rise to asymmetric peak profiles.

The limit of detection of aerosol MALDI as it is now implemented is not as low as for surface MALDI: more material is consumed over more laser shots for aerosol MALDI. It is possible to estimate the total material consumed for each spectrum and the amount of sample present in the interaction volume during a single laser pulse. The total amount of material flowing into the mass spectrometer for UV ionization can be estimated from the solution concentration and flow rate and from the instrument geometry. For the spectrum shown in Figure 3 , the bovine insulin concentration was $3.4 \times 10^{-4} \mathrm{M}$ and the liquid flow rate was 8 $\mu \mathrm{L} / \mathrm{s}$. This corresponds to a transfer rate of $3 \mathrm{nmol} / \mathrm{s}$ of bovine insulin to the exit of the nebulizer. Note that at this flow rate the quantity of bovine insulin con sumed for the mass spectrum in Figure 3 was 600 nmol. The fraction of the aerosol beam transported into the ionization chamber can be estimated from the divergence of the aerosol beam, the nozzle-to-skimmer distance, and the skimmer tube diameter. With the gcometry used in this cxperiment, approximatcly $20 \%$ of the aerosol passes through the drying tube into the ionization chamber; the remainder is deposited on the walls of the nebulizer chamber. The collimated aerosol beam that passes into the ionization chamber is $5 \mathrm{~mm}$ in diameter, nearly an order of magnitude larger than the $0.5-\mathrm{mm}$ diameter focused laser beam. This geome- 
try gives a $10 \%$ overlap between the aerosol and laser beams. The resulting total efficiency of transport of material from the nebulizer capillary to the irradiated volume is thus approximately $2 \%$. Viewed in terms of quantity of analyte, $60 \mathrm{pmol}$ of bovine insulin flow through the interaction volume each second. Because the laser is pulsed, only a small fraction of these molecules is irradiated. The pulse width of the UV laser is $6 \mathrm{~ns}$, during which time approximately 0.3 amol ( $2 \times 10^{5}$ molecules) of bovine insulin is irradiated. An estimate of the number of ions formed in one laser pulse can be obtained from the area-under-thebovine-insulin ion peaks. The average integrated bovine insulin transient signal using the $50 \Omega$ input of the digital oscilloscope is $6 \times 10^{-9} \mathrm{Vs}$. Using the manufacturer's specified gain of $10^{7}$ for the microchannel plate detector yields a total charge of $1 \times 10^{-17} \mathrm{C}$ (approximately 100 ions). This calculation indicates that approximately one out of every 2000 irradiated analyte molecules is ionized.

The optical microscopy results indicate that at least some of the aerosol particles sent through the drying tube still contain solvent. The protonated methanol clusters in the low mass portion of the bovine insulin aerosol MALDI mass spectrum further indicate the presence of solvent in the aerosols. These clusters are formed along with the positively charged analyte, suggesting that photon absorption causes a breakup of the aerosol particle into solvent containing clusters. The absence of positively charged matrix ions indicates that the matrix molecules are either neutral, negatively charged, or if positively charged, present in only large particles or clusters. The presence of matrix ions observed in negative ion mode indicates that at least some of the matrix molecules acquire a negative charge (Murray, K. K.; Beeson, M. D.; Russell, D. H.; unpublished results). Negatively charged methanol clusters are not observed. A possible explanation for the observed ions is that clusters are formed by the breakup of the irradiated aerosol particles. Consider a particle containing matrix $A H$ and $n$ solvent molecules $S$ :

$$
\text { Aerosol particle }+h \nu \rightarrow S_{n} \cdot[A H]^{*}
$$

The notation $[A H]^{*}$ indicates a matrix molecule with excess electronic or vibrational energy. The matrix molecule may donate a proton to the cluster:

$$
[A H]^{*} \cdot S_{n} \rightarrow A^{-} \cdot H^{+} \cdot S_{n}
$$

and dissociate to form a cluster ion pair:

$$
A^{-} \cdot H^{+} \cdot S_{n} \rightarrow A^{-} \cdot S_{n-m}+H^{+} \cdot S_{m} \text {. }
$$

Although highly speculative, this mechanism is consistent with the fact that matrix negative ions are observed and matrix positive ions are not, whereas only positively charged methanol clusters are observed.
The results shown in Figure 6 show that the area and width of the bovine insulin $\mathrm{M}^{+}$peak increase as the temperature of the drying tube is decreased. At lower drying tube temperatures, the amount of solvent in the aerosol particles should be higher. This is consistent with the peak width data in that more solvent in the aerosol particle leads to a greater degree of solvent-analyte clustering and a broader analyte $\mathrm{M}^{+}$ peak. The fact that the area of the $\mathrm{M}^{+}$peak increases with increasing aerosol particle solvation indicates that the solvent is promoting total analyte ion formation, possibly by limiting fragmentation (see below). Our experience is that the longer drying tube gives a good analyte ion signal even at low temperature, whereas a short drying tube (or none at all) gives a poor analyte signal at all temperatures. This suggests that contact time with the drying gas in the drying tube is more important than the drying tube temperature. This assessment is consistent with the observation of $50-\mu \mathrm{m}$ aerosol particles with a $6-\mathrm{cm}$ drying tube and $10-\mu \mathrm{m}$ particles with a $25-\mathrm{cm}$ drying tube, whereas the particle diameter did not vary with drying tube temperature in either case.

One of the most striking differences between aerosol and surface MALDI is the optimum matrix-to-analyte ratio. Surface MALDI typically uses a $10^{2}$ to $10^{4}$ molar excess of matrix, whereas aerosol MALDI is optimized at the lowest possible ratio. This effect has been noted previously [26] and is ascribed to differences in crystal formation for the two methods. Typical surface MALDI samples are formed when a solution containing the matrix and analyte slowly evaporates on a metal probe tip at ambient temperature and pressure. Analyte molecules present at low concentrations in the solution are included in the matrix crystal that forms as the solution slowly evaporates. Beavis and Bridson [7] found that proteins preferentially bind to certain crystal faces of the MALDI matrix trans-sinapic acid (trans3,5-dimethoxy-4-hydroxy-cinnamic acid) through hydrophobic interactions. This being the case, there must be a minimum number of matrix molecules required to form a crystal face suitable for inclusion of a single analyte molecule. After one analyte molecule is included, a large number of additional matrix molecules will be necessary to surround the included analyte and form another crystal face. At a matrix-to-analyte ratio below an optimum value, the additional analyte will not be included in the matrix and the crystallization and inclusion process may in fact be hindered.

The aerosol sample forms when the matrix and analyte-containing droplets are rapidly desolvated in vacuum. Aerosol particles in the nebulized spray quickly lose solvent and possibly even freeze due to evaporative cooling. The aerosol particles are heated in the drying tube to drive off additional solvent and create a dry particle. In this "freeze-drying" process, it is possible that the dried aerosols do not have large crystal domains or appreciable long range order. The analyte may be randomly distributed in the aerosol 
particle and thus in direct contact with the matrix molecules. In such a solid, a matrix-to-analyte ratio close to unity will lead to the largest number of ions because each analyte molecule is in contact with a matrix molecule. To summarize the argument, the rapidly freeze-dried aerosols lock the matrix and analyte into favorable positions as the solvent is driven off the particle. The slowly air-dried crystals of conventional MALDI favor a large matrix-to-analyte ratio for optimum analyte inclusion.

Another significant difference between aerosol and surface MALDI is the high laser irradiances that can be used with the former. Irradiances in excess of 1 $\mathrm{Gw} / \mathrm{cm}^{2}$ can be used with no observable analyte fragmentation. Note that because the laser pulse width and spot size are held constant, the pulse energy, fluence, and irradiance are proportional. A possible explanation for the tolerance of aerosol MALDI for high irradiance is the presence of solvent in the aerosol. Energy deposited in the aerosol particle can be removed by solvent evaporation as the droplet breaks up. Furthermore, solvent containing clusters formed on aerosol particle breakup can continue to release excess energy by solvent evaporation.

\section{Instrument Improvements}

The major limitations for aerosol MALDI as an analytical technique are limit of detection and resolution. The resolution of the aerosol MALDI mass spectra shown above is 6 , a factor of $\mathbf{5 0}$ lower than the resolution typically observed for surface MAI.DI. The factors limiting resolution that can be most easily addressed experimentally are ion spatial distribution, ion source region pressure, and aerosol solvation. TOF mass resolution can be influenced by spatial, temporal, and kinetic energy distribution of ions. Ion spatial distribution is usually neglected in surface MALDI because ionization can be assumed to take place on a surface of negligible thickness. With aerosol MALDI ions are formed across the width of the laser beam and this leads to the initial ion spatial distribution. The time required for an ion to traverse the length of the TOF-MS is given by [27]

$$
\iota=\left(\frac{m}{E e s_{0}}\right)^{1 / 2}\left[D+\sqrt{2} s_{0}\right]
$$

where $m$ is the mass of the ion of charge $e$ formed in a field of gradient $E$. The ion is formed a distance $s_{0}$ from the extraction grid and traverses a field free flight tube of length $D$. For simplicily the initial ion kinelic energy has been set to 0 and complete ion formation is assumed at $t=0$. The width of the laser beam leads to a distribution of $s_{0}$ values and results in a temporal spreading of the ion arrival signal.

The influence of ion spatial distribution on resolution can be minimized in two ways. First, the focus of the laser beam can be improved to reduce the laser beam diameter at the point of intersection with the aerosol beam. Second, an additional acceleration region can be added. The configuration of the aerosol MALDI apparatus is essentially that of a WileyMcLaren TOF-MS [28]. The electric fields in two acceleration regions can be adjusted to give a spatial focus at the detector. Additionally, time lag focusing can be employed to compensate for the initial energy spread of the ions [28].

High ion source region pressure can cause poor resolution because of collisions in the acceleration region. The pressure in the ion source region can be improved with greater pumping speed or reduced gas load. It is likely that the nebulizer and drying tube can be improved to provide more efficient desolvation and a greater aerosol flow with a reduced carrier gas flow. An ultrasonic nebulizer can also be used to create a monodisperse aerosol with particles of approximately $10 \mu \mathrm{m}$ in diameter, smaller than are created with the pneumatic nebulizer and more easily desolvated. More efficient desolvation of the aerosols can also be expected to limit clustering between the solvent and analyte ions and thus reduce the widths of the analyte peaks.

The limit of detection for aerosol MAI.DI can be improved by optimizing aerosol transport efficiency, aerosol-laser beam overlap, laser duty cycle, and ionization efficiency. As stated above, the current aerosol transport efficiency is $2 \%$ and thus up to a factor of 50 improvement can be realized with changes in the design of the nebulizer and skimmer/drying tube. The aerosol-laser beam overlap is maximized when the two beams have the same diameter. Mass resolution is maximized, however, when the spatial extent of the ions along the flight path is minimized (eq 2). The optimum configuration is a narrow aerosol beam and a laser bcam narrow along the flight axis and wide along the aerosol beam axis. A cylindrical lens can produce such a laser focus. Laser duty cycle can be improved with existing technology. The maximum repetition rate for a commercially available LV laser is $500 \mathrm{~Hz}$ for an excimer laser, and the 308- or 351-nm excimer UV wavelengths are suitable for aerosol MALDI applications. The laser duty cycle can improve the limit of detection by a factor of 200 (25-ns pulse width at 500 $\mathrm{H} z$ versus 6-ns pulse width at $10 \mathrm{~Hz}$ ). The high pulse energy of the excimer is ideal for cylindrical focusing: a $200-\mathrm{mJ}$ pulse can be focused to $3 \mathrm{~cm} \times 0.02 \mathrm{~cm}$ and still result in an irradiance of $100 \mathrm{~mJ} / \mathrm{cm}^{2}$. This will improve the limit of detection by a factor of 60 . Ionization efficiency should improve with decreased aerosol size and increased aerosol drying efficiency. An ionization efficiency of unity corresponds to an improvement in the limit of detection of 2000 .

Taken together, the above instrument improvements show that it may be possible to increase the limit of detection of aerosol MALDI by as much as $10^{9}$. At this level of detection, the 600 nmol consumed in 
acquiring the mass spectrum in Figure 3 is reduced to $600 \mathrm{amol}$. The time for data acquisition changes from 3 minutes at $10 \mathrm{~Hz}$ to $4 \mathrm{~s}$ at $500 \mathrm{~Hz}$. Although highly speculative at this early stage of instrument development, the potential analytical utility of aerosol MALDI is apparently quite high.

A potentially important application for aerosol MALDI is as a detector for HPLC separations. The liquid flow rate used in aerosol MALDI is compatible with HPLC separations that typically have flow rates between 0.5 and $1 \mathrm{~mL} / \mathrm{min}$ (10 to $20 \mu \mathrm{L} / \mathrm{s}$ ). Aerosol MALDI mass spectra of the quality shown in Figures 3 and 5 can be obtained in 1000 to 2000 laser shots and acceptable mass spectra can be obtained in 100 laser shots. At a $10-\mathrm{Hz}$ repetition rate a complete mass spectrum can be acquired every $10 \mathrm{~s}$, adequate for HPLC separations with peaks in the range of 50 to 100 s. At $500 \mathrm{~Hz}$, comparable mass spectra could be obtained for peaks with widths of 1 to $2 \mathrm{~s}$.

\section{Conclusions}

MALDI has been successfully applied to aerosols. Aerosol MALDI shows promise as a technique to investigate the MALDI process on consistent and continuously produced aerosol particle surfaces, and as a means to couple liquid separation methods with MALDI mass analysis. Aerosol MALDI mass spectra are similar in general appearance to those obtained with surface MALDI. Specific differences for aerosol MALDI are (1) lower mass resolution; (2) lower limit of detection; (3) low mass region ( $<300 \mathrm{Da}$ ) dominated by solvent clusters; 4) low optimum matrix-toanalyte molar ratio; (5) high optimum laser irradiance. Instrument improvements have been outlined for improvement of mass resolution and detection limit. The tolerance for high liquid flow rates and the potential for rapid TOF acquisition of entire mass spectra make aerosol MALDI a potentially useful interface for LC/MS. Preliminary results with HPLC separation and postcolumn matrix introduction indicate that aerosol MALDI can be used as a detection method in the present configuration of the apparatus [29]. Improvements in limit of detection will make this technique even more useful.

\section{Acknowledgments}

The authors thank Randy Scott and the Texas A\& M Electron Microscopy Center for access to the optical microscope. Funding for this research was provided by the Texas Advanced Technology Program and by the Division of Chemical Sciences, Office of Basic Energy Sciences, U.S. Department of Energy under grant no. DE-FG05-85ER-13434. The Nd:YAG laser and additional equipment were purchased with funds provided by the National Science Foundation under grant no. CHE-8821780.

\section{References}

1. Karas, M.; Bachman, D.; Bahr, U.; Hillenkamp, F. Int. J. Mass Spectrom. Ion Processes 1987, 78, 53-68.

2. Tanaka, K.; Waki, H.; Ido, Y.; Akita, S.; Yoshida, Y. Rapid Commun. Mass Spectrom, 1988, 2, 151-153.

3. Karas, M.; Hillenkamp, F. Anal. Chem. 1988, 60, 2299-2301.

4. Hillenkamp, F.; Karas, M; Beavis, R. C.; Chait, B. T. Anal. Chem. 1991, 63, 1193A-1202A.

5. Overberg, A.; Karas, M.; Bahr, U.; Kaufmann, R.; Hillenkamp, F. Rapid Communt. Mass Spectrom. 1990, 4, 293-296.

6. Strupat, K.; Karas, M.; Hillenkamp, F. Int. I. Mass Spectrom. Ion Processes 1991, 111, 89-102.

7. Beavis, R. C.; Bridson, J. N. J. Phys. D. 1993, 26, 442-447.

8. Overberg, A.; Karas, M.; Hillenkamp, F. Rapid Commun. Mass Spectrom. 1991, 5, 128-131.

9. Strobel, F. H.; Solouki, T,; White, M. A.; Russell, D. H. J. Am Soc. Mass Spectrom. 1991, 1, 1-4.

10. Zhao, S.; Somayajula, K. V.; Sharkey, A. G.; Hercules, D. M.; Hillenkamp, F.; Karas, M.; Igendoh, A. Anal. Chem. 1991, 63, 450-453.

11. Chan, T.-W.; Colburn, A. W.; Derrick, P. J. Org. Mass Spectrom. 1992, 27, 53-56.

12. Li, L.; Wang, A. P. L.; Coulson, L. D. Anal. Chem. 1993, 65, 493-495.

13. Lustig, D. A.; Lubman, D. M. Rev. Sci. Instrum. 1991, 62, 957-962.

14. Köster, C.; Dey, M.; Grotemeyer, J.; Schlag, E. W. Praceedings of the 38th Annual ASMS Conference on Mass Spectrometry and Altied Topics; Tucson, AZ, 1990; pp 1242-1243.

15. Wang, A. P. L.; Li, L. Anal. Chem. 1992, 64, 769-775.

16. Nelson, R. W.; Thomas, R. M.; Williams, P. Rapid Commun. Mass Spectrom. 1990, 4, 348-351.

17. Williams, E. R.; Jones Jr., G. C.; Fang, L.; Nagata, N.; Zare, R. N. In Applied Spectroscopy in Materials Science II; W. G. Golden, Ed.; Proc. SPIE 1636, 1992; pp 172-181.

18. McKeown, P. J.; Johnston, M. V.; Murphy, D. M. Anal. Chem. 1991, 63, 2069-2073.

19. Sinha, M. P. In Applied Spectroscopy in Materials Science; D. D. Saperstein, Ed.; Proc. SPIE 1437, 1991; pp 150-156.

20. Murray, K. K.; Russell, D. H. Anal. Chent. 1993, 65, 2534-2537.

21. Håkansson, P.; Kamensky, I.; Sundqvist, B.; Fohlman, J.; Peterson, P.; McNeal, C. J.; Macfarlane, R. D. J. Am. Chem. Soc. 1982, 104, 2948-2949.

22. Barber, M.; Bordoli, R. 5.; Elliot, G. J.; Sedgwick, R D.; Tyler, A. N.; Green, B. N. J. Chem. Soc. Chem. Commur. 1982, 936-938.

23. Buko, A. M.; Phillips, L. R.; Fraser, B. A. Biomed. Mass Spectrom. 1983, 10, 408-419.

24. Barber, M.; Bordoli, R. S.; Elliot, G. J.; Tyler, A. N.; Bill, J. C.; Green, B. N. Biomed. Mass Spectrom. 1984, 11, 182-186.

25. Gimon, M. E.; Preston, L. M.; Solouki, T.; White, M. A.; Russell, D. H.; Org. Mass Spec. 1992, 27, 827-830.

26. Murray, K. K.; Russell, D. H. Proceedings of the Second International Conference on Laser Ablation, April 19-22, 1993, Knoxville, TN (in press).

27. Cotter, R. J. Anal. Chem. 1992, 64, 1027-1039A.

28. Wiley, W. C.; McLaren, I. H. Reo. Sei. Instrum. 1955, 26, 1150-1157.

29. Murray, K. K.; Russell, D. H. Procedings of the 41st Annual ASMS Conference on Mass Spectrometry and Allied Topics; San Francisco, CA, 1993; p 780a. 